\title{
3 Research Square

\section{The retinal lesions in ultra-wide-field retinal imaging and the consistency of different fundus screening methods in HIV/AIDS patients}

\section{Kuifang Du}

Capital Medical University Youan Hospital

\section{Chao Chen}

Capital Medical University Youan Hospital

\section{Chungang Guo}

Capital Medical University Youan Hospital

\section{Hongwei Dong}

Capital Medical University Youan Hospital

\section{Lian-yong Xie}

Capital Medical University Youan Hospital

\section{Wenjun Kong}

Capital Medical University Youan Hospital

Wen Bin Wei ( $\nabla$ tr_weiwenbin@163.com )

https://orcid.org/0000-0001-7983-8241

\section{Research article}

Keywords: HIV, AIDS, Ultra-wide-field retinal imaging, retinopathy, screening

Posted Date: October 15th, 2019

DOI: https://doi.org/10.21203/rs.2.16051/v1

License: (a) (1) This work is licensed under a Creative Commons Attribution 4.0 International License. Read Full License 


\section{Abstract}

Background: To observe the retinal lesions in HIV/AIDS patients and to evaluate the consistency of nonmydriatic ultra-wide-field (UWF) retinal imaging and mydriatic slit lamp biomicroscope with Superfield lens.

Methods: 193 eyes of 98 consecutive HIV/AIDS patients were enrolled. The retinal lesions in each patients were observed through UWF fundus imaging and slit lamp biomicroscopic with Superfield lens whose consistency was analyzed.

Results: 100 eyes (51.8\%囚 had fundus lesions, 20 eyes (20\%) presented posterior microvascular retinopathy (MVR), 19 eyes (19\%) presented peripheral MVR, 15 eyes (15\%) presented early stage cytomegalovirus retinitis (CMVR), 6 eyes showed initial stage CMVR, and 51 eyes showed other changes (papilledema, etc.). The consistency of two methods was moderate in detecting the isolated peripheral lesions (Kappa $=0.445)$ or HIV-related MVR (Kappa $=0.513)$, and high in inspecting the posterior/posterior involved lesions (Kappa $=0.831)$ or CMVR $(\mathrm{Kappa}=1.0)$. The detection rate of UWF retinal imaging and slit lamp biomicroscopic were 15.5\% $\varangle 30 / 193 \rrbracket$ and $17.6 \% \varangle 34 / 193 \rrbracket(P=0.557 \rrbracket$ 0.05) for HIV-related MVR, 7.8\% (15/193) ( $P=1.000 \bowtie 0.05)$ for CMVR, 37.3\% (72/193) and 33.7\% (65/193) $(P=0.118 \otimes 0.05)$ for posterior/posterior involved lesions, 8.8\% (17/193) and 17.6\% (34/193) $(P=0.001 \rrbracket$ $0.05)$ for isolated peripheral lesions, respectively.

Conclusions: The HIV-related MVR can be posterior or peripheral. Various fundus changes appear in HIV/AIDS patients, not only MVR or CMVR. The non-mydriatic UWF fundus imaging system and mydriatic slit lamp biomicroscope exhibited good consistency and nondiscriminatory detection rate for CMVR, HIVrelated MVR and posterior lesions, but not for isolated peripheral lesions.

\section{Background}

The current estimated national HIV prevalence rate in China are $0.0598 \%$ according to he HIV/AIDS data from the national surveillance systems of China ${ }^{[1]}$. Once an infectious retinitis appeared in AIDS patients, lesions would rapidly progress, causing irreversible vision loss or blindness. Nearly $19.7 \%$ to $44.25 \%$ of HIV/AIDS patients had fundus damages ${ }^{[2-3]}$. In the Post-HAART-Era, the most common fundus opportunistic infection is still cytomegalovirus retinitis (CMVR), which is also known as the main cause of vision loss in these patients. In fact, some CMVR patients who are detected in early stage or without macular involvement, is treatable and can thus avoid blindness. Therefore, it is crucial for HIV/AIDS patients to conduct routine ocular screening and regular follow-up so as to detect early lesions and reduce visual impairment ${ }^{[4]}$. Traditionally, the most commonly adopted method for fundus screening was mydriatic fundoscopy with indirect ophthalmoscopy (90D microscope lens and 20D indirect lens, Superfield lens, etc.), or one field digital fundus photography comprising $30^{\circ}$ to $45^{\circ}$ posterior pole. A research had confirmed that the traditional fundus digital photography has a low sensitivity $(30.2 \%)$ in the telemedicine screening for CMVR. The missed CMVR lesions were more likely to be small and located 
in the peripheral retina. The author suggested that an improved accuracy would require a camera which is able to image the peripheral retina more easily ${ }^{[5]}$.

The ultra-wide-field (UWF) fundus imaging systems: Optos ${ }^{\circledR}$ ( Optos Carfornia ${ }^{\circledR}$, Optos PLC, Dunfermline, United Kingdom) is a pioneer in UWF fundus imaging systems. Due to the advantages of wide field, nonmydriatic, convenient and time-saving operation, UWF was widely used and was designed to cover up to $200^{\circ}$ (about $80 \%$ ) of the retina in a single image, thereby making the image information more complete, and enhancing our understanding of peripheral retinal diseases ${ }^{[6]}$.

This analysis aimed to observe the fundus change of HIV/AIDS patients via UWF fundus imaging systems, which might bring us new discoveries. Moreover, the consistency between the non-mydriatic UWF fundus imaging systems and mydriatic slit lamp biomicroscope was analyzed.

\section{Methods}

Approved by Ethics Committee of Beijing Youan Hospital, Capital Medical University (LL2018150K), this prospective observational study targeted 193 eyes of 98 consecutive HIV/AIDS patients from the ophthalmology department of Beijing Youan Hospital between October and December 2018.

Patients who were previously diagnosed with HIV infection or AIDS, with or without ocular symptoms were enrolled in this study. Patients should voluntarily accept these ocular examinations, including non-mydriatic UWF fundus photography, mydriasis medication and slit lamp biomicroscope with Superfield lens. Exclusion criteria: poor image quality; serious opacity of refractive media.

All the enrolled patients received routine ophthalmological examinations, such as visual acuity, intraocular pressure and slit lamp. The basic information (age and gender) and a recent blood CD4+T lymphocytes level were collected. A trained member (Dr. Du) took all fundus photographs during the study with Optos UWF fundus imaging systems under the natural state of pupil. After the shooting, all eyes had compound tropicamide eye drops every 5 minutes for a total of 3 times. After the pupils dilated, a seasoned retina specialist (Dr. Chen) subsequently checked each eye using indirect ophthalmoscopy with Superfield lens (Volk Optical, USA) but was not aware of the findings of the fundus photography. The type and location of fundus lesions were recorded independently to avoid potential sources of bias. The lesions were recorded at the posterior pole or peripheral part according to the position of vortexes vein. Patients with poor image quality were excluded from the study. If a fundus disease involved posterior pole or peripheral part simultaneously, we classified it into the posterior/posterior involved lesions. If different fundus diseases appeared in the same eye, we recorded these fundus diseases separately. When the diagnoses of two approaches were inconsistent, another retina specialist (Dr. Xie) rechecked the same eye with indirect ophthalmoscopy combining UWF fundus photography or optical coherent tomography (ОCT). All clinical information and blood test results were available for Dr. Du, Dr. Chen and Dr. Xie. 
All the diagnoses in this study were based on fundus examination. CMVR was defined through fundus examination showing local or quadrantal retinal necrosis: cheese ketchup necrosis, granular appearance or frosted branch retinal angiitis. CMVR was divided into two categories as follows: (1) initial stage (when the focus is less than one disc diameter and observation was approximately less than one week after onset), (2) early stage (when the initial therapeutic dose of anti-CMV agents is used and the observation was approximately less than 2 to 4 weeks after onset) ${ }^{[7]}$. For HIV-related MVR, a participant was defined as having posterior or peripheral cotton wool spots, microaneurysms, telangiectasia, or retinal hemorrhages.

SPSS 25.0 software was adopted for statistical analysis. The results were recorded according to the presence or absence of the lesions detected by each method. The consistency Statistics (Kappa value) was performed to determine the agreement between two approaches. The kappa value was standardized to range from -1 to 1 scale, where 1 is perfect agreement and 0 represents what would be expected by chance. Negative values indicate the potential disagreement between the observers. Positive values make sense. The larger the Kappa value is, the better the consistency is. The consistency statistics where kappa values were considered as: 1 , perfect; $1.0-0.75$, good; $0.75-0.40$, moderate; $₫ 0.40$, poor. The differences were assessed in age and $\mathrm{CD}+4 \mathrm{~T}$ lymphocyte between some fundus lesions through Kruskal-Wallis test. Cross tabulation and the chi-square test were applied to compare the rate of diseases with two screening methods. $P$ values $<0.05$ were regarded as statistically significant.

\section{Results}

Patient characteristics

193 eyes of 98 HIV/AIDS patients were confirmed eligible in our study, including 93 males $(94.9 \%)$ and 5 females (5.1\%). UWF images of three eyes were excluded because of serious vitreous opacity or cataract. The mean patient age was $37.40 \pm 10.58$ years (range $21-76$ yeas). The mean blood CD $4+T$ lymphocyte, which were available in 93 patients, was $167.22 \pm 237.72 \mathrm{cells} / \mu \mathrm{l}$ (range $2-1089 \mathrm{cells} / \mu \mathrm{l})$. The blood CD4+T lymphocytes was less than 200 cells/ $\mu$ in 71 patients $(72.45 \%)$, more than 200 cells $/ \mu$ in 22 patients $(22.45 \%)$, and unclear in 5 patients $(5.10 \%)$.

Fundus changes

Among the $98 \mathrm{HIV/AIDS}$ patients, 33 patients (33.7\%) were normal while 65 patients (66.3\%) had fundus changes. There were 93 eyes (48.2\%) with normal fundus and 100 eyes (51.8\%) with fundus changes. 20 eyes $(20 / 193,10.4 \%)$ manifested posterior MVR, 19 eyes (19/193, 9.8\%) presented peripheral MVR, 15 eyes $(15 / 193,7.8 \%)$ showed CMVR, and 51 eyes had other fundus changes (such as papilledema, peripheral lattice degeneration, PORN, diabetic retinopathy, etc.) (Table 1). Some eyes may present two or more different lesions simultaneously. In addition to the conventional changes, some new lesions were discovered: peripheral MVR, refers to peripheral microaneurysms, telangiectasia, retinal hemorrhages, but no cotton wool spots was found (Figure 1); initial stage CMVR, refers to posterior or peripheral small granular lesions about 1-3 disc-diameter, which is similar to granular CMVR (Figure 2). These 
similar retinal changes were divided into four groups: Group 1: Posterior MVR; Group 2: Peripheral MVR; Group 3: Early stage CMVR; Group 4: Initial stage CMVR. No significant difference was found in age nor CD4+T lymphocyte $(P>0.05)$ between those groups.

No adverse events were found during our study.

Consistency check

In general, these two approaches showed moderate consistency in the detection of isolated peripheral lesions (Kappa value $=0.445$ ), and high consistency in the detection of posterior/posterior involved lesions (Kappa value $=0.831>0.75$ ). The consistency of two approaches in detecting HIV-related microvascular retinopathy was moderate as well(Kappa value $=0.513)$, and it was completely consistent for CMV retinitis (Kappa value $=1.0>0.75)$ (Table 2$)$.

\section{Detection rate}

The detection rate of UWF fundus imaging and slit lamp biomicroscope were 15.5\%区30/193囚and $17.6 \% \varangle 34 / 193 \rrbracket(P=0.557 \rrbracket 0.05)$ for HIV-related MVR, 7.8\% (15/193) $(P=1.000 \otimes 0.05)$ for CMVR, 37.3\% $(72 / 193)$ and $33.7 \%(65 / 193)(P=0.118 \otimes 0.05)$ for posterior/posterior involved lesions, $8.8 \%(17 / 193)$ and $17.6 \%(34 / 193)(P=0.001 \otimes 0.05)$ for isolated peripheral lesions, respectively (Table 3$)$.

\section{Discussion}

Besides HIV-related MVR and CMVR, different fundus changes were found in HIV/AIDS patients. HIVrelated MVR could be posterior or peripheral. These CMVRs in initial stage have not been placed much emphasis in clinic and rarely reported in literature. These findings yielded new insights for our future research to understand the significance and possible long-term consequences. It was also confirmed that the UWF fundus imaging system and Superfield lens showed good consistency and nondiscriminatory detection rate for CMVR, HIV-related MVR and posterior lesions, thereby laying a scientific basis to our future clinical work.

Non-contact Daytona UWF fundus imaging system in this study provides $200^{\circ}$ range of retinal imaging, and brings great convenience for ophthalmic clinic. A large number of studies have been conducted on the observation of peripheral retinal conditions of fundus diseases through UWF laser scanning ophthalmoscopy ${ }^{[8]}$, aiming to reduce the burden of ophthalmic outpatient service by combining with optical coherence tomography for virtual retinal outpatient service ${ }^{[9]}$. With UWF fundus imaging, we confirmed that the most common HIV-related fundus changes were HIV-related MVR, followed by CMVR, which met the results of another group's study ${ }^{[3,10]}$. Other changes included HIV related/non-related lesions: optic disc edema, peripheral lattice degeneration, subretinal lesions, and PORN, etc. HIV-related MVR refers to non-infectious retinal vascular changes. The diagnosis was based on the findings of microaneurysms, telangiectasia, retinal hemorrhages, and cotton wool spots (CWS) ${ }^{[11]}$. One assumption regarding the pathogenesis of HIV/AIDS-related retinopathy is that HIV-1 can induce an inflammatory 
state in human retinal pigment epithelial (HRPE) cells, which results in impairment of HRPE barrier function ${ }^{[12]}$. The symptoms were not aroused by these lesions, and were usually discovered through routine fundus eye screening. The prevalence of HIV-related microvascular retinopathy was $2 \%$ in patients with CD 4+T lymphocytes $\geq 200$ cells $/ \mu \mathrm{l}, 15 \%$ in patients with CD $4+T$ lymphocytes $<200$ cells $/ \mu \mathrm{l}$. The CD4+T lymphocytes was $<200$ cells/ $\mu$ in $87 \%$ of patients with HIV-related microvascular retinopathy ${ }^{[10]}$. Traditionally, it is believed that HIV-related MVR lesions are located at the posterior pole. However, we found 2 types of HIV-related MVR with both UWF fundus imaging and traditional fundoscopy: Type 1: Cotton wool spots at the posterior pole, with slight retinal hemorrhage; Type 2: Peripheral retinal microangioma, telangiectasias or patchy superficial retinal hemorrhage. The prevalence of type 2 was similar to that of type 1 (19/193 VS 20/193). There was also no difference in age or CT4+T lymphocyte level between these groups. The peripheral retinal vascular abnormalities are novel findings in HIV/AIDS patients. Like our awareness of the peripheral microvascular lesions in diabetic retinopathy through UWF fundus imaging ${ }^{[13-14]}$, further studies are needed to understand the significance and possible long-term consequences of these findings.

As HIV-related MVR lesions are small and scattered, especially in some patients with thick nerve fiber layer around optic disc, the CWS is easy to be ignored in UWF fundus imaging. These lesions are displayed more clearly under high magnification of the slit lamp biomicroscope with Superfield lens. The detection method may also miss these small CWS lesions due to the narrow light band especially when patients were sensitive to light stimulation and could not cooperate with eye rotation. Although a small number of missing diagnosis of HIV-related MVR will not affect the treatment plan, it could affect the evaluation of patients' condition, as some patients may further develop CMVR ${ }^{[15]}$. Therefore, strict ophthalmic follow-up should be carried out for these patients clinically. Although both examination methods have their limitations, the statistical results showed a moderate level of consistency and similar detection rate with no significant differences. For peripheral lesions, it brings challenges to both screening methods. Due to the distortion of peripheral retinal images, eyelid/eyelash shielding and the high reflection point from eyelashes in UWF fundus imaging system, it is difficult to obtain real peripheral microvascular lesions. We also found that the consistency of the two methods for isolated peripheral lesions was worse than that for the posterior retinopathy, with the Kappa value $=0.445$ and 0.831 , respectively. The detection rate for isolated peripheral lesions with UWF fundus imaging system was also significantly lower than slit lamp biomicroscope ( $8.8 \%$ vs $17.6 \%)$, indicating that mydriatic slit lamp biomicroscope with Superfield lens or UWF fundus photograph combining different eye position should be recommended for peripheral fundus screening.

6 patients in group 4 was classfied as initial stage CMVR. This lesion constitutes several well-defined white granules, about 1-3 disc-diameter. It is distinct in appearance from the early stage CMVR or MVR. In the optical coherence tomography, these white granular lesions showed concavity of the retinal nerve fber layer (RNFL) toward the retinal pigment epithelium and destruction of all retinal layers vertically; while the cotton wool spot showed elevation of the RNFL and no destruction of the retinal structure ${ }^{[7]}$. We divided it into an independent group and compared it with early stage CMVR group, posterior MVR group 
and peripheral MVR group in age and CD4+T lymphocyte level. However, no difference was found. More information should be collected from HIV/AIDS patients, such as the use of HAART, blood/aqueous CMVDNA level and HIV viral load, which are also the limitations in this study.

The diagnosis of CMVR was based on clinical signs and symptoms. If not treated in time, the disease will turn to total retinal necrosis and retinal detachment, thereby leading to irreversible vision loss. The UWF Optomap imaging system captured greater areas of total retina and peripheral CMVR lesions than conventional fundus photography, and was more favored by patients because of the perceived time requirements and comfort of use ${ }^{[16]}$. For CMVR patients without receiving HAART, it is recommended to begin HAART after the induction period of systemic anti-cytomegalovirus treatment, which is beneficial to reduce the possibility of immune reconstruction of uveitis ${ }^{[17]}$. Intravitreal injection of antiviral drug is widely used: ganciclovir or foscarnet sodium (mostly adopted for drug-resistant strains) ${ }^{[18]}$. Therefore, it is necessary to conduct CMVR screening before starting HAART for high-risk patients (such as patients with low income level, long distance to hospital, systemic symptoms, CMV-DNA positive blood, patients who have not received HAART treatment and CD4+T<200 cells/ $\left.\mu{ }^{[10,15,19]}\right)$. CMVR screening can not only clarify the fundus condition, but also guide the treatment plan. In this study, we found that two common fundus screening methods for HIV/AIDS patients were completely consistent with the same detection rate. because the reason is that CMVR has a wide range of lesions and obvious characteristics, and is not easy to be ignored in UWF fundus photograph nor Superfield lens. So, we can choose either method to provide these CMVR screening for HIV/AIDS patients.

However, we were unable to carry out further research related to the peripheral MVR, or initial stage CMVR in this study as the aqueous humor, vitreous specimens or other important variable were not obtained from these patients. Although the machine operation and fundus examination in this study were conducted by seasoned ophthalmologists independently, there may be potential bias.

\section{Conclusion}

The HIV-related MVR can be posterior or peripheral. There are various fundus changes in HIV/AIDS patients, not only MVR or CMVR. The non-mydriatic UWF fundus imaging system and mydriatic slit lamp biomicroscope showed good consistency and nondiscriminatory detection rate for CMVR, HIV-related MVR and posterior lesions, but not for isolated peripheral lesions.

\section{Abbreviations}

UWF, ultra-wide-field; CMV: Cytomegalovirus; MVR: microvascular retinopathy; CMVR, CMV retinitis; PORN, Progressive outer retinal necrosis; DR, diabetic retinopathy; PVR, proliferative vitreoretinopathy

\section{Declarations}

Ethics approval and consent to participate: 
The study protocol of the present study was approved by the Ethics Committee of Beijing Youan Hospital, Capital Medical University (LL-2018-150-K), and informed consent was obtained from all patients. The informed consent obtained from every patients was written.

Consent for publication:

Not applicable.

Availability of data and material:

The datasets used and/or analysed during the current study available from the corresponding author on reasonable request.

Competing interests:

The authors declare that they have no competing interests.

Funding:

This study was supported by Scientific Research Project of Beijing Youan Hospital, CCMU, 2018 \YNKTQN20180201).

Authors' contributions:

KFD analysed the data and drafted the manuscript. CC and LYX collected the data and revised the manuscript. CGG, HWD and WJK performed the study and revised the manuscript. WBW designed and performed the study, collected the data and gave final approval of the version to be published. All authors read and approved the final manuscript.

Acknowledgements:

Not applicable.

\section{References}

[1]. Ming-Bo H, Li Y, Bing-Yu L, et al. Characterizing the HIV/AIDS Epidemic in the United States and China[J]. International Journal of Environmental Research and Public Health, 2015, 13(1):30-.

[2]. Chiotan C, Radu L, Serban R, et al. Posterior segment ocular manifestations of HIV/AIDS patients[J]. Journal of medicine and life, 2014, 7(3):399-402.

[3]. Kim Y S, Sun H J, Kim T H, Kang K D, Lee S J. Ocular Manifestations of Acquired Immunodeficiency Syndrome. Korean Journal of Ophthalmology: KJO. 2015;29(4):241-248. 
[4]. Ausayakhun S, Yen M, Jirawison C, et al. Visual acuity outcomes in cytomegalovirus retinitis: early versus late diagnosis. British Journal of Ophthalmology 2018;102:1607-1610.

[5]. Jirawison C, Yen M, Leenasirimakul P, et al. Telemedicine Screening for Cytomegalovirus Retinitis at the Point of Care for Human Immunodeficiency Virus Infection. JAMA Ophthalmol. 2015;133(2):198-205.

[6]. Nicola Quinn, Lajos Csincsik, Erin Flynn, Christine A. Curcio, Szilard Kiss, SriniVas R. Sadda, Ruth Hogg, Tunde Peto, Imre Lengyel,The clinical relevance of visualising the peripheral retina. Progress in Retinal and Eye Research. 2019;68:83-109.

[7]. Shigeko Yashiro, Takeshi Nishijima, Yuuka Yamamoto, Yumi Sekine, Natsuyo YoshidaHata, Tomohiro lida, Shinichi Oka. Spectral domain optical coherence tomography and fundus autofuorescence fndings in cytomegalovirus retinitis in HIVinfected patients.Japanese Journal of Ophthalmology (2018) 62:373389.

[8]. Nivison-Smith L, Milston R, Chiang J, Ly A, Assaad N \& Kalloniatis M. Peripheral retinal findings in populations with macular disease are similar to healthy eyes. Ophthalmic Physiol Opt 2018; 38: 584-595.

[9]. Lee JX, Manjunath V, Talks SJ. Expanding the role of medical retina virtual clinics using multimodal ultra-widefield and optical coherence tomography imaging. Clin Ophthalmol. 2018:12 Pages 2337-2345

[10]. Nishijima T, Yashiro S, Teruya K, et al. Routine Eye Screening by an Ophthalmologist Is Clinically Useful for HIV-1-Infected Patients with CD4 Count Less than $200 / \mu \mathrm{L}[\mathrm{J}]$. Plos One. 2015, 10(9):e0136747.

[11]. Michael W. Stewart. Human immunodeficiency virus and its effects on the visual system. Infect Dis Rep. 2012 Mar 8;4(1):e25.

[12]. Tan S, Duan H, Xun T, et al. HIV-1 impairs human retinal pigment epithelial barrier function: possible association with the pathogenesis of HIV-associated retinopathy[J]. Laboratory Investigation, 2014, 94(7):777-787.

[13]. Kiss, Szilárd, Berenberg T L. Ultra Widefield Fundus Imaging for Diabetic Retinopathy[J]. Current Diabetes Reports, 2014, 14(8):514.

[14]. Nivison-Smith, Lisa Milston, Rebecca Chiang, Jaclyn Ly, Angelica Assaad, Nagi Kalloniatis, Michael. Peripheral retinal findings in populations with macular disease are similar to healthy eyes. Ophthalmic and Physiological Optics. 2018, 38. 584-595.

[15]. Chen C, Guo CG, Meng L, et al. Comparative analysis of cytomegalovirus retinitis and microvascular retinopathy in patients with acquired immunodeficiency syndrome. Int J Ophthalmol. 2017;10(9):13961401. 
[16]. Mudvari S S, Virasch V V, Singa R M, et al. Ultra-wide-field imaging for ytomegalovirus retinitis.[J]. Ophthalmic Surgery Lasers \& Imaging the Official Journal of the International Society for Imaging in the Eye, 2010, 41(3):311-5.

[17]. French M A, Meintjes G. Immune Reconstitution Disorders in Patients with HIV Infection[J]. Infectious Diseases (Fourth Edition). 2017, 2: 859-864.

[18]. Becker K N, Becker N M. Ocular manifestations seen in HIV [J]. Dis Mon. 2014, 60(6):268-275.

[19]. Leenasirimakul P, Liu Y, Jirawison C, et al. Risk factors for CMV retinitis among individuals with HIV and low CD 4 count in northern Thailand: importance of access to healthcare[J]. British Journal of Ophthalmology.2016, 100(8):1017.

\section{Tables}

Table 1: Classification of the fundus lesions

\begin{tabular}{lll}
\hline Pathological type & Affected eyes & Percentage \\
\hline Posterior MVR & 20 & $10.4 \%$ \\
\hline Peripheral MVR & 19 & $9.8 \%$ \\
\hline Early stage CMVR & 15 & $7.8 \%$ \\
\hline Papilledema & 11 & $5.7 \%$ \\
\hline Peripheral lattice degeneration & 10 & $5.2 \%$ \\
\hline DR & 6 & $3.1 \%$ \\
\hline Initial stage CMVR & 6 & $3.1 \%$ \\
\hline Drusen & 4 & $2.1 \%$ \\
\hline PORN & 4 & $2.1 \%$ \\
\hline Old retinal lesions & 4 & $2.1 \%$ \\
\hline Subretinal lesion & 3 & $1.6 \%$ \\
\hline Subretinal hemorrhage & 2 & $1.0 \%$ \\
\hline Tractional retinal detachment & 1 & $0.5 \%$ \\
\hline rhegmatogenous retinal detachment & 1 & $0.5 \%$ \\
\hline PVR & 1 & $0.5 \%$ \\
\hline Normal & 93 & $48.2 \%$ \\
\hline
\end{tabular}

Table 2: The consistency of UWF fundus imaging system and slit lamp biomicroscope

\begin{tabular}{lll}
\hline Pathological type & Kappa value & Consistency \\
\hline Isolated peripheral lesions & 0.445 & Moderate \\
\hline Posterior/posterior involved lesions & 0.831 & Good \\
\hline HIV-related microvascular retinopathy & 0.513 & Moderate \\
\hline CMVR & 1.0 & Perfect \\
\hline
\end{tabular}

Table 3ロThe detection rate of UWF fundus imaging and slit lamp biomicroscope 
HIV-related

MVR
CMVR Posterior

/posterior involved lesions
Isolated

peripheral

lesions

\section{UWF}

$15.5 \%$

$7.8 \%$

$37.3 \%$

$8.8 \%$

slit lamp

$17.6 \%$

$7.8 \%$

$33.7 \%$

$17.6 \%$

biomicroscopy

$\chi^{2}$ values

51.154

$193.00 \quad 133.960$

44.492

$\mathrm{P}$ values

0.557

1.000

0.118

0.001

\section{Figures}

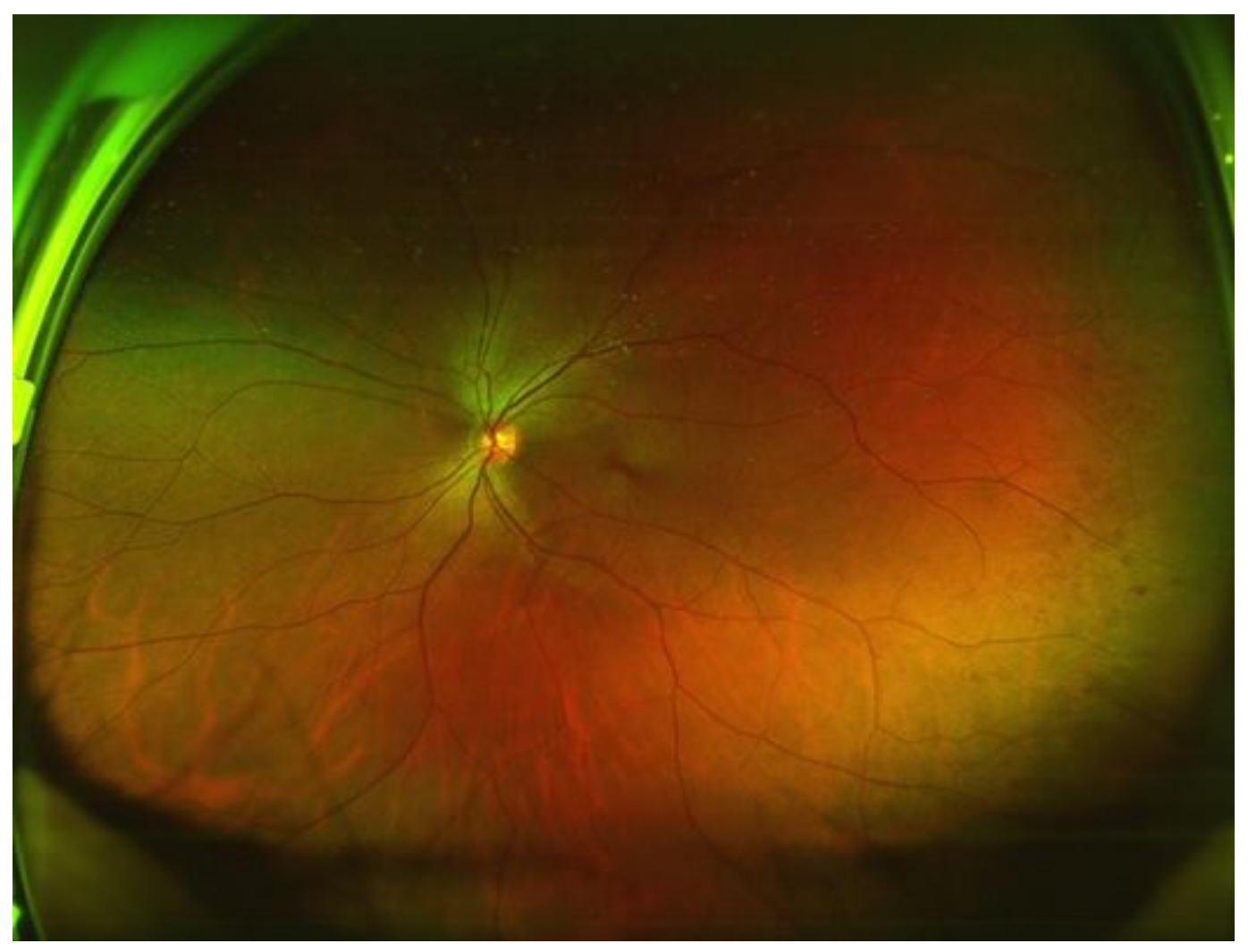

Figure 1 
Ultra-wide-field fundus photograph of peripheral MVR with peripheral microaneurysms

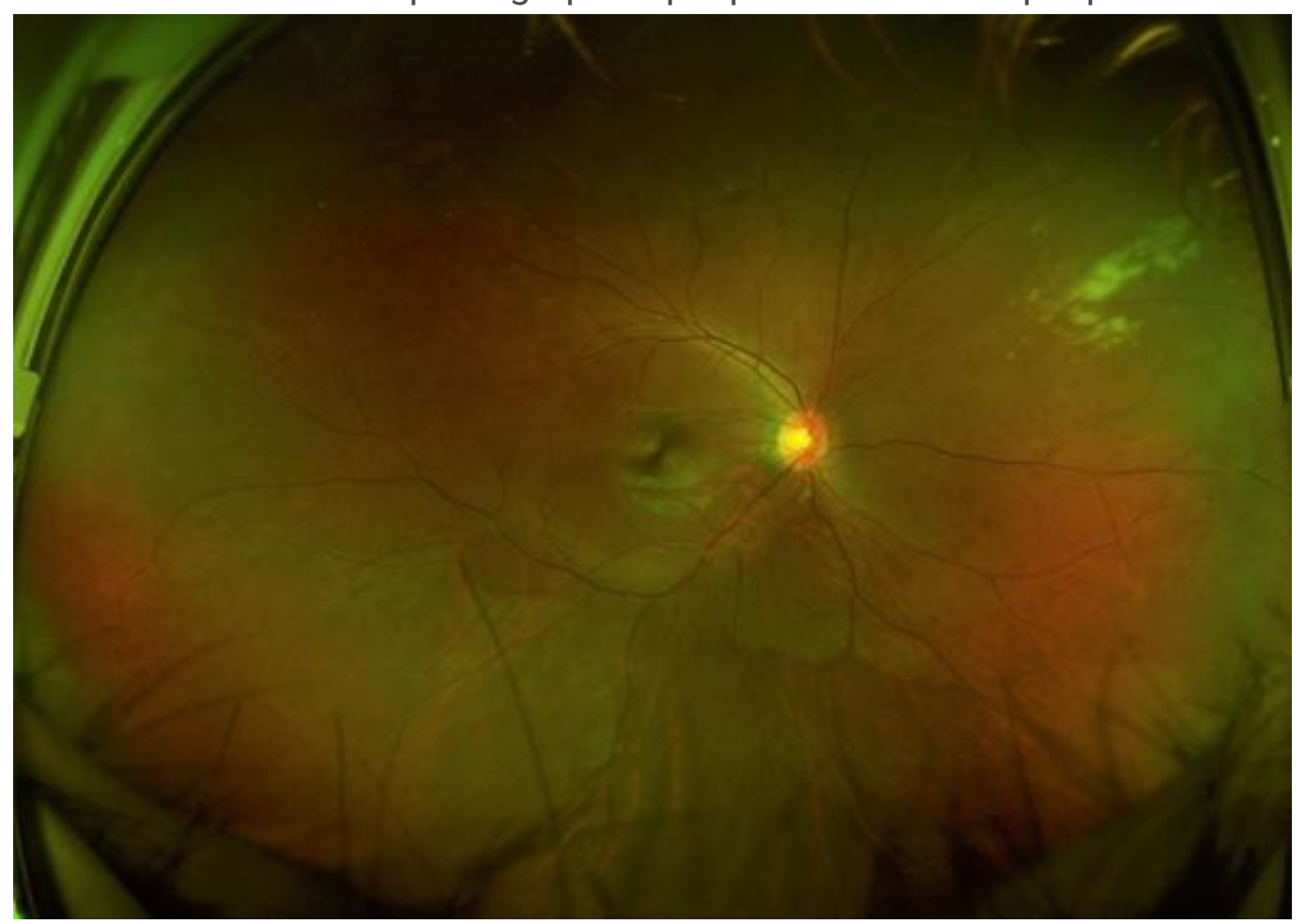

\section{Figure 2}

Ultra-wide-field fundus photograph of peripheral MVR with peripheral microaneurysms 